CENTRO TEDESCO DI STUDI VENEZIANI

Venetiana

Collana diretta da Sabine Meine

12 



\section{Venezia e Dalmazia}

a cura di

Uwe Israel e Oliver Jens Schmitt

viella 
(C) 2013 Viella s.r.l. - Centro Tedesco di Studi Veneziani, Roma-Venezia Prima edizione: maggio 2013

ISBN 978-88-6728-010-0

Volume pubblicato con i contributi della Fondazione Fritz Thyssen per la Promozione delle Scienze, dell'Institut für Osteuropäische Geschichte, Università di Vienna, e del Delegato del Governo Tedesco per la Cultura e i Media - Fritz Thyssen Stiftung für Wissenschaftsförderung, Institut für Osteuropäische Geschichte, Universität Wien, und Beauftragter der Bundesregierung für Kultur und Medien aufgrund eines Beschlusses des Deutschen Bundestages.

Cura redazionale di Michaela Böhringer

\section{viella}

libreria editrice

via delle Alpi, 32

00198 ROMA

www.viella.it
Centro Tedesco di Studi Veneziani

Palazzo Barbarigo della Terrazza

S. Polo 2765/A - Calle Corner

30125 Venezia

www.dszv.it 
Indice

Uwe Israel e Oliver Jens Schmitt

Premessa

ERMANNo ORLANDO

Politica del diritto, amministrazione, giustizia.

Venezia e la Dalmazia nel basso medioevo

TOMISLAV RAUKAR

La Dalmazia e Venezia nel basso medioevo

Oliver Jens Schmitt

Storie d'amore, storie di potere:

la tormentata integrazione dell'isola di Curzola

nello Stato da mar in una prospettiva microstorica

Francesco Bettarini

Il notariato dalmata e la "Santa Intrada"

Josip VRANDEČIĆ

La Dalmazia nell'età moderna:

L'influsso della "rivoluzione militare" sulla società dalmata 



\section{Oliver Jens Schmitt}

Storie d'amore, storie di potere.

La tormentata integrazione dell'isola di Curzola

nello Stato da mar in una prospettiva microstorica

Il 13 maggio 1444 Marco Gradenigo, onorabile conte di Curzola, sedente sulla loggia «ad bancum iuris» insieme con ser Stanoje Obradović, giudice minore, e ser Marin Žilković, nonché ser Giacobbo Criacobi, ambedue giudici maggiori, condannò un certo Zuanin Dragačić, figlio di Francesco, a sei mesi in carcere e a una ammenda di 200 perperi, perché Dragačić aveva violentato Franuša, la figlia di Radašin da Stagno. Franuša aveva partorito una «puerulla» che aveva poi affidato a un prete, don Luca, che la portò a Stagno, dove la battezzò e la consegnò a una balia. Il padre della ragazza che aveva intentato l'azione contro Dragačić ottenne piena soddisfazione. Siccome Dragačić non si presentò davanti ai giudici, fu condannato in contumaciam, una procedura che pareva pienamente giustificata. ${ }^{1}$

Un caso tragico per la ragazza coinvolta e la sua famiglia, certo, ma i registri di «criminalia» di Curzola non sono poveri di processi di stupro e di violenza sessuale. Un caso normale quindi, uno tra tanti altri, furti, lesioni corporali, il rifiuto di un contadino di dare l'allarme di pirati, che furono esaminati dai giudici lo stesso giorno del 13 maggio? Se non ci fosse una piccola nota in margine del registro, niente ci farebbe pensare altrimenti, ma la nota suscita la curiosità dello studioso: «il 23 giugno 1445 questa sentenza fu cassata in accordo con lettere ducali ricevute lo stesso giorno e registrate negli atti di cancelleria».

Il caso è stato portato davanti alle autorità centrali a Venezia e supera dunque la dimensione strettamente locale, un caso di rilievo sociale, forse anche politico. Questa ipotesi diventa certezza, quando si studia l'atto

1. Državni arhiv u Zadru (Archivio storico di Zara). Arhiv Korčule (Archivio di Curzola) (= DAZ, AK); tutte le citazioni di fonti archivistiche si riferiscono a questo archivio. 10/13/1, f. 47v-56r. 
completo del processo: parecchie decine di documenti si riferiscono alle dramatis personae. Ciò che ci si offre è un caso classico di microstoria che ci permette di ricostruire le vicende biografiche di una coppia di innamorati nel suo contesto sociale, e che ci permette di esplorare nuove dimensioni della presenza veneziana in Oltremare alla fine del medioevo. L'archivio di Curzola, che conserva serie complete di processi penali e che è unico nel suo genere in tutta la Dalmazia veneziana, apre anzi alla ricerca delle possibilità nuove e affascinanti. ${ }^{2}$

Siccome questo contributo non si limita a un semplice racconto dei fatti, ma intende metterli in un contesto più ampio, ci pare utile dividerlo in sei passi.

Il primo passo ricostruisce il processo; il secondo fornisce uno schizzo del contesto politico generale dell'isola di Curzola verso la metà del XV secolo; il terzo analizza le principali persone coinvolte; il quarto passo sarà consacrato all'analisi delle forme e dei modi di conflittualità; il quinto mette in rilievo il ruolo di Venezia come potere politico reale, come ombra e discorso; infine ritorneremo ai nostri protagonisti narrando brevemente il loro percorso biografico ulteriore.

1. Il 7 agosto 1444, Michele Michiel, nuovo conte di Curzola, interrogò di nuovo Franuša, citata su richiesta di Zuanin Dragačić, che la accompagnò al tribunale. ${ }^{3}$ Franuša ritrattò tutto quello che aveva attestato qualche mese prima e narrò una storia del tutto diversa: che Dragačić e lei sarebbero da tre anni una coppia di innamorati; che mai il suo amante avrebbe usato violenza contro di lei; che avrebbe partorito non uno, ma due figli, di cui uno era morto; che sarebbe riuscita a tenere segreta la relazione a suo padre e che Dragačić non sarebbe stato il suo primo amante. Poi spiega gli antecedenti del processo contro Dragačić. Suo padre e il maestro Giorgio Grupšić l'avrebbero forzata ad accusarlo; sarebbe stata picchiata da suo fratello e che solo sua sorella Katuša avrebbe tentato, invano, di correre in suo soccorso. Altri testimoni confermano e completano l'immagine.

2. Foretić, Otok Korčula u srednjem vijeku do g. 1420 e Dokoza, Dinamika otočnog prostora si occupano del periodo pre-veneziano. Id., Prilog proučavanju unutrašnjih prilika na otoku Korčuli u srednjem vijeku; Id., Obrambeni sustav Korčulanske komune u srednjem vijeku; Schmitt, Korčula sous la domination. Foretić, Borbe između pučana i plemića na Korčuli u 15. i 16. stoljeću presenta un'analisi degli scontri politici a Curzola, senza fare uso del materiale dell'archivio di Curzola, ma basandosi soprattutto sui suoi Statuti.

3. DAZ, AK, 10/15/3, f. 97r-v. 
Antonio Stanojević si mostra ben informato: conosce le chiacchiere sulla coppia, ha sentito, dalla bocca di maestro Giorgio, della nascita di un figlio, della morte dell'altro; sa che un certo Alessio di Antonio aveva visto il conte Gradenigo che stava leggendo negli statuti di Curzola e aveva esclamato: «Questo capitolo è contro Zuanin Dragačić che ha una relazione con la figlia di Radašin da Stagno». ${ }^{4}$

Ascoltiamo un altro testimone, Pribislav da Krajina, originario dunque della terraferma dalmata e che si rivela il postillon d'amour di Franuša, intimo conoscitore di tutti i dettagli di questa storia d'amore: durante una notte d'inverno, notte di pioggia e neve, notte d'allarme per paura di pirati catalani, gli uomini si adunarono sulla piazza; in quella notte il testimone stava passando davanti alla casa di Radašin, quando Franuša lo chiamò e lo pregò di far venire Zuanin; Pribislav dichiara di averle prestato molte volte lo stesso servizio, invitando l'amante nella casa della ragazza quando quella era sola. Risulta da questo racconto che Franuša aveva preso l'iniziativa e che sapeva con molta astuzia sottrarsi al rigido controllo sociale nella piccola città di Curzola. L'ultimo testimone aggiunge alcuni dettagli sugli antecedenti di Franuša e il predecessore di Dragačić nel suo letto, il fabbro Dabiživ e Zuanin Grupšić, figlio di Giorgio, cioè dell'uomo che stava dietro il processo, un ragazzo conosciuto per aver aspramente litigato con il suo successore a causa di Franuša. Un caso classico di riscatto e di vendetta, dunque. Ma cosa c'entrano il conte e la sua lettura degli statuti? Perché l'appello arriva fino al doge Francesco Foscari?

L'enigma si risolve quando si allarga l'analisi e si conosce il motivo dell'assenza di Dragačić. Questi stava negoziando proprio con il doge nella sua funzione di rappresentante incaricato dei contadini di Curzola; Dragačić fu nient'altro che il leader incontestato dei contadini curzolani nel loro conflitto con i patrizi, un uomo che godeva dell'appoggio e della fiducia del grande doge, che fece subito cassare la sentenza.

2. Abbiamo raggiunto il livello politico e sociale del nostro processo, e per dar senso a questo caso di microstoria occorre schizzare il quadro in cui essa va inserita. Nell'anno 1420, la Repubblica di Venezia e i patrizi di Curzola conclusero un contratto che regolava l'integrazione dell' isola nello Stato da mar. Questo contratto segue nelle sue linee principali le tradizioni ben note della politica espansionista della Serenissima nel bacino adriatico: 
riconoscimento del diritto locale, cioè degli statuti, del sistema politico già esistente, degli offici, delle proprietà, elezione di un conte veneziano da parte dei patrizi locali. ${ }^{5}$ L'interesse della Signoria si spiega con i vantaggi strategici che offriva la piccola isola; in immediata vicinanza con la grande concorrente di Ragusa, Curzola forniva alla navigazione veneziana il primo porto sicuro dopo Durazzo, una posizione chiave all'entrata del mondo isolano dalmata. Ma quello che per la Signoria era un accordo di routine, costituiva a Curzola un vero pomo della discordia: i patrizi che avevano firmato il contratto a nome dell'isola intera non rappresentavano tutti gli abitanti; avevano trascurato i diritti e le rivendicazioni dei contadini che non si sentivano soggetti alla città. Questi possedevano i loro propri consigli, chiamati veće, e erano abituati a decidere i loro affari politici a livello locale. A differenza di Zara o di Sebenico, dove i patrizi erano riusciti a eliminare l'autonomia delle comunità rurali, una simile pretesa di patrizi curzolani è rimasta lettera morta di fronte a un mondo rurale orgoglioso e anche violento nella difesa dei suoi diritti. Lo squilibrio tra la pretesa politico-sociale dei patrizi e una realtà curzolana sensibilmente diversa creava una tensione enorme tra patrizi e contadini. Incapaci di imporre la loro volontà nello spazio rurale, i primi cercavano alleati, trovandoli nei ranghi dei cittadini di Curzola-città e, in alcuni casi, anche nei loro omologhi, i patrizi veneziani che detenevano la funzione di conte a Curzola; questo era il caso di Marco Gradenigo. È dunque fuori dubbio che il processo contro Dragačić fu intentato non da maestro Giorgio Grupšić solo, ma con l'appoggio attivo del conte e di un gruppo di patrizi che miravano alla rovina della carriera politica e dell'esistenza sociale del loro avversario, Zuanin Dragačić. Raccontiamo, solo a margine, che un altro processo contro lo stesso personaggio esaminava il furto di una capra, che Dragačić e i suoi compagni, già in viaggio verso Venezia, avrebbero rubato a un pastore - anche questa una trappola, visto che era uso a Curzola comprare degli animali sul pascolo, pagando il pastore e non il proprietario; ma quando Dragačić, una volta salito sulla nave, chiamò il pastore, questi si nascose nella macchia; il suo comportamento si spiega sapendo che il proprietario era un patrizio, nemico giurato di Dragačić che voleva criminalizzarlo come ladro comune.

3. Arriviamo al terzo capitolo, l'analisi delle dramatis personae che allarga anche l'orizzonte cronologico del nostro percorso narrativo. Co- 
minciamo con il nostro «eroe» o più esattamente con i nostri eroi, perché abbiamo a che fare con due fratelli, Zuanin e Zanin, che non soltanto dal nome si assomigliavano come due gocce d'acqua. Figli di Francesco e di Gojca, nipoti di Marino, ${ }^{6}$ sono nati, probabilmente verso il 1420, a Čara, villaggio nel cuore dell'isola. Appaiono per la prima volta nei registri dell'amministrazione veneta verso la fine degli anni Trenta, e, diciamolo già in questa fase del nostro racconto, riempiono gli stessi registri fino alla loro morte, Zanin scomparendo nel 1458, mentre Zuanin invece visse almeno fino al 1476. ${ }^{7}$ Per quanto ne sappiamo, loro padre Francesco fu un contadino medio, senza aspirazioni politiche. Quando i fratelli sorgono dall'ombra dei documenti, dimostrano già alcune qualità di carattere che distingueranno il loro percorso ulteriore: ${ }^{8}$ si resero nemici mortali in un processo di eredità contro il patrizio Marco Goriglavić (a partire del 1438); nel 1441 li vediamo coinvolti in un conflitto serio con il ceto patrizio; a quest'epoca, i contadini preparavano una delegazione a Venezia per difendere i loro diritti contro i nobili, e dunque l'agitazione sociale fu considerevole: il conte temeva l'entrata di «multi incoli in magna comitiva»e la proibì sotto la pena di quindici giorni in carcere e la confisca di un terzo del possesso immobile, pena esagerata che lascia sentire l'inquietudine del magistrato. ${ }^{9}$ Il 27 settembre il conte proibì a Dragačić di uscire dalla città - «non audeat neque presumat recedere a civitate sine litera quam judices mitere volebant ad Serenissimum ducale dominium», ${ }^{10}$ divieto al quale questi rispose con parole minacciose, piene di disprezzo. Il giorno dopo, Dragačić tenne un discorso davanti a una folla di contadini e quando un patrizio, membro della potentissima famiglia di Obradović, passò di lì con i suoi seguaci, e Dragačić lo provocò al duello («nolite venire super me omnes, sed veniat unus vestrum qui velit ad pugnandum mecum» ${ }^{11}$ ); il

6. DAZ, AK, 20/20/1, f. 24r: Zuanin de quondam Francesco Marinovich.

7. DAZ, AK, 25/48/18, 2 f. 50r.

8. Nell'agosto 1440 , i due fratelli appaiono come testimoni in un atto di procura per il loro futuro avversario, ser Forte d'Antonio (DAZ, AK, 8/11/1, f. 309v); nell'ottobre dello stesso anno, incontriamo ser Forte e Zanin Dragačić come testimoni, di nuovo in un atto di procura (ivi, f. 344v).

9. DAZ, AK, 7/1071, f. 36v «et hoc quia magnus eror et dissensiones in civitate occurrere posint, presente ser Antonio quondam ser Bartoli et maistro Benedicto Laurentii de Veneciis testibus ... statuens dictis vilicis quod quicquid facere in civitate intendunt sive procuratores sive alias scripturas quod hodie per totam diem eorum facta expedire debeant».

10. DAZ, AK, 7/1071, f. 36v.

11. DAZ, AK, 7/10/1, f. 37r. 
nobile rifiutò e si ritirò pieno di vergogna. Nel dicembre dello stesso anno i patrizi contrattaccarono, accusando Dragačić di aver violato gli statuti di Curzola perché aveva difeso i diritti di un forestiero. La fase successiva del conflitto cominciò nel 1444, quando in primavera Dragačić partì per Venezia $^{12}$ e i suoi nemici invece preparavano il colpo mortale che abbiamo già descritto. Riprendiamo il filo del nostro racconto: nell'agosto del 1444 Dragačić e i suoi compagni tornarono da Venezia, una novità che si divulgò rapidamente sull'isola; il conte incaricò il giudice nobile Giovanni Mikšić di arrestarlo nel suo villaggio natale di Čara; Mikšić incontrò nel cammino il guardiano Michele Radomilić, già avvertito da sua moglie che Dragačić aveva adunato i suoi seguaci, tutti armati fino ai denti. Il giorno fu drammatico: Dragačić, un arco nella mano, 12 frecce nella faretra, il tredicesimo nell'altra mano, suo fratello con una spada, 12 guardiani del corpo (uomini che avevano giurato di difendere Dragačić fino alla morte) e dietro di loro tutto il villaggio - tutti aspettavano il giudice. Questi esortò i contadini a consegnare Dragačić, ma solo una piccola minoranza di quattro uomini si accinse a obbedire - che si ritirarono subito quando videro l'arco di Dragačić e le facce dei suoi seguaci. Il giudice prese subito la fuga, ben felice di tornare sano e salvo a Curzola-città.

Anche dopo il fallimento della cospirazione del conte e del gruppo radunato attorno a Grupšić, i patrizi non disarmarono; ma neanche Dragačić cedette di un passo. Tutte e due le parti portarono il loro caso davanti alle autorità centrali a Venezia; i patrizi furono rappresentati da Forte d'Antonio, membro di una famiglia italiana immigrata a Curzola, ricco proprietario, uomo d'affari con investimenti in Puglia e nei Balcani, capo di una rete di contrabbandieri, uomo politico potente. In assenza di Dragačić, la caccia a Franuša fu ripresa: il 20 gennaio 1445 fu emanato il divieto di aiutarla di allontanarsi dall'isola, pena l'ammenda enorme di 200 perperi; poco dopo fu emessa un'altra grida che obbligava gli isolani a denunciare il nascondiglio di Franuša su pena di due mesi di carcere; il 19 febbraio fu effettuata una perquisizione di una casa fuori le mura di Curzola da Radašin, il padre di Franuša, dal «plazarius» (guardia campestre) e da alcuni uomini incaricati del conte; volevano già partire, quando trovarono un fazzoletto di Franuša, che riuscì a scappare; i poliziotti confiscarono il suo guardaroba, «una gonela di pavonazo di valore di 8 ducati, una tovalia magna a tabula

12. I popolani gli avevano dato una «commissione» il 30 settembre 1443, DAZ, AK, 10/14/1, f. 160r. 
di valore di 2 ducati, un fustagno, 9 fazoli a mano novi di valore di $2 \mathrm{du}$ cati...», che assommava oggetti di valore per 18 ducati - una bella fortuna per una ragazza (la dote di una ragazza contadina ammontava all'epoca da 3 fino a 6 ducati). ${ }^{13} \mathrm{E}$ chi aveva nascosto la ragazza? La famiglia i cui diritti i Dragačić avevano difeso nel processo di eredità già accennato. Il ritorno di Dragačić, in tasca la cassazione della sentenza contro di lui, pose fine subito a quest'episodio del conflitto, ma solo in questa fase.

Le strutture del conflitto sembrano chiare, una lotta di patrizi contro contadini; ma uno sguardo più attento alle fonti rivela delle costellazioni sociali più complesse. Cominciamo con i patrizi: bisogna distinguere tra patrizi nella città e patrizi che risiedevano nello spazio rurale - uno di quelli che volevano arrestare Dragačić a Čara apparteneva a questa seconda categoria; nell'interrogatorio del conte attestò che era continuamente escluso dai consigli contadini e dunque anche emarginato dagli abitanti del villaggio che gli celavano i loro "segreti". I patrizi nemici di Dragačić appartenevano in prima linea alle famiglie Obradović, Žilković e d'Antonio; ma sarebbe erroneo attribuire loro una omogeneità o unità d'azione, una strategia politica comune o il pensare in categorie di segregazione sociale assoluta. Il caso di Giorgio Grupšić è emblematico; questo cittadino - dunque non patrizio - nutriva un odio privato contro Dragačić, ma la vendetta personale non sembra aver motivato esclusivamente le sue azioni politiche; Grupšić combatteva quello che percepì come disordine sociale, cioè le adunanze dei contadini, le loro delegazioni a Venezia, le loro negoziazioni con il doge; era pronto a sostenere ogni conte che procedeva contro i contadini e accompagnò Forte d'Antonio a Venezia dove ottenne, il 19 gennaio 1446, un ducale di Francesco Foscari in favore della loro causa: ${ }^{14}$ ma questo non significa che fosse un soggetto leale della Serenissima: quando il successore di Gradenigo applicò altri ducali in favore di Dragačić, Grupšić dimostrò una ostilità aperta contro il dominio veneziano. Nel luglio 1449 la flotta veneziana vinse una battaglia contro i Catalani di Alfonso d'Aragona; in questa occasione Grupšić derise alcuni Curzolani che festeggiavano la vittoria, dicendo che il Re di Napoli e il suo alleato, Giovanni Hunyadi, avrebbero fra poco conquistato tutta l'Istria e la Dalmazia, osando dire in faccia del patrizio veneziano Pasquale Gradenigo «m'alora la signoria ha pigliato guera cum re e Ragona perché s'el Re de Ragona farà liga cum 
Vaivoda Ivanis de Ungaria per Dio piglarà tuta Dalmatia e Istria, el corpo de Veniexia» ${ }^{15}$ - Dragačić, informato di questo incidente, lo denunciò subito, cosa che valse a Grupšić un processo di alto tradimento al quale si sottrasse fuggendo per sempre dall'isola. Fu il trionfo di Dragačić; il nipote di Grupšić, invece, Zuanin Grupšić, rimase sull'isola e non perse il suo stato sociale (Dragačić l'aiutò contro suo zio Giorgio in un processo per una vigna $\left.{ }^{16}\right)$. Grupšić fu l'esponente più radicale del ceto conservatore, non composto solo da patrizi.

Il padre di Franuša, invece, Radašin da Stagno, non può essere annoverato tra questo gruppo; egli era immigrato dal territorio di Ragusa, e sperava di radicarsi nella società curzolana prestando dei servizi sporchi al ceto patrizio. Dichiarava di essersi stabilito a Curzola già da bambino. Da uomo modesto, si lasciò ingannare dai patrizi, che lo strumentalizzarono per i loro intrighi politici. Aveva due figlie, Franuša e Katuša e alcuni figli maschi che appaiono nei registri della cancelleria veneziana; nel giugno 1449, Božić, fratello di Franuša, fu arrestato di notte, perché era salito con un compagno sulle mura per portare una cassetta a Ragusa; alle guardie dichiarò di aver portato solo un pezzo di formaggio, che apparteneva a Franuša, alla porta di mare $\cdot{ }^{17}$ Radašin disponeva di una piccola fortuna che gli permise, nel 1446, il viaggio a Venezia, e, nel 1448, di comprare una nave. ${ }^{18}$ Morì prima del 1459. ${ }^{19}$

Nel novembre 1445, Forte d'Antonio lanciò un altro attacco contro Dragačić: un gruppo di cittadini di Curzola gli rimproverano di parlare ingiustamente a nome di tutti gli strati popolani dell'isola; questo gruppo si chiamava «zitadini del povolo antigo» e rappresentava gli artigiani residenti nella città. Significava dunque che la frattura sociale seguiva il contrasto città-spazio rurale? La risposta è negativa, primo a causa dei patrizi rurali, secondo perché questi «zitadini del povolo antigo» non sembrano costituire la maggioranza dei non-patrizi nella città. Il pomo della discordia erano le rivendicazioni politiche dei contadini e delle loro comunità ruraliche rivendicavano il diritto di riunione - privilegio tradizionale, come sottolineavano - le adunanze, le veće, le quali, agli occhi dei patrizi, erano riunioni illegali, veri nidi di sedizione sociale. Nella sua risposta all'accusa

15. DAZ, AK, 13/22/3, f. 305v.

16. DAZ, AK, 12/19/2, f. 115 r 4 agosto 1449.

17. DAZ, AK, 11/1718, f.42v. 6 giugno 1449.

18. DAZ, AK, $9 / 12 / 1$, f. $68 \mathrm{r} ; 8 / 11 / 2$ f. $226 \mathrm{v}$.

19. DAZ, AK, $15 / 28 / 3$, f. 122 r-v. 
di Forte d'Antonio, Dragačić riassume la storia dei diritti contadini, invoca i nomi dei conti veneziani, Pancrazio Zorzi, Tommaso Michiel, Marco Gradenigo, che avevano permesso tali adunanze le quali erano sempre convocate al suono della campana nella chiesa di San Pietro, più tardi nella chiesa di Ognissanti a Curzola-città secondo «antiga usanza e consueto»; tali adunanze erano destinate a comporre $\mathrm{i}$ «capituli» o petizioni dei contadini che si sarebbero presentati al Senato veneziano. Dragačić stesso spiegò nel 1462 al conte, Antonii «mi habiando stà contra questi zintilhomeni tante volte a Veniecia e stato caxon de frige (?) tuor la libertà de la election dei rectori principalmente de le man de ser Mathio e so fradelli li quali erano quelli li qual solu eligivano per esser de mazor parenti de Curzula», ${ }^{20}$ riferendosi alla dinastia degli Obradovići, il cui monopolio di potere egli aveva distrutto.

Curzola non faceva eccezione nelle tensioni sociali che caratterizzano tutta la Dalmazia tardo-medievale, ma come abbiamo già sottolineato, costituisce un'eccezione perché i contadini opponevano una resistenza molto più efficace al discorso egemonico dei patrizi. Le strutture dei gruppi rivaleggianti si capiscono solo quando ci si rende conto del fatto che la lotta di potere non fu decisa nel XV secolo, ma che continuò ininterrottamente fino alla fine del medioevo e anche oltre. Questo fatto spiega anche il comportamento talvolta contraddittorio di alcuni protagonisti che furono costretti a trovare modi di convivenza con i loro avversari. L'isola e la città erano piccole, i protagonisti del conflitto politico-sociale si incontravano spesso nelle strade, sulla piazza, al porto; 1'ascesa sociale e economica dei Dragačići, la crescita della loro potenza economica obbligò anche membri del patriziato a scendere a compromessi, soprattutto nel mondo degli affari. Nel 1448, Zuanin Dragačić, il conte Marco Soranzo e Mateo Obradović furono visti al porto, dove conclusero un contratto di vendita di vino; aumentando il suo capitale, Dragačić riuscì ad affittare la dogana di Curzola ${ }^{21}$ ed in questa funzione forniva il prodotto più ricercato nei Balcani ai mercanti patrizi della dinastia Obradović (a Francesco), vale a dire il sale. Alcuni patrizi come Marin Baronić appaiono come avvocati o procuratori, insieme con Zuanin, creando in tal modo una rete di interessi comuni. Baronić diventò un partner importante di Zuanin; partner d'affari, ma anche conciliatore in processi con altri patrizi. A prima vista, l'atteggiamento dei patrizi in un processo politico del

20. DAZ, AK, 17/32 3, f. 30r.

21. DAZ, AK, 12/13/1, f. 35v per la somma di 140 ducati. 
1448 potrebbe stupire: Dragačić aveva accusato il conte Soranzo di aver abusato della sua funzione nel processo contro di lui nell'affare Franuša; Marino Baronić, ma anche Forte d'Antonio, interrogato da Dobroslav Obradović l'uomo che Dragačić aveva umiliato sette anni prima - e altri patrizi attestarono che il conte aveva manipolato il processo, aveva messo sotto pressione i giudici e che si era comportato non da giudice, ma da accusatore. Nel 1456, Frana Obradović nominò Zuanin tutore dei suoi figli; ${ }^{22}$ nello stesso anno, Dragačić entrò a far parte dell'opposizione nobile contro la decima sugli animali, che il nuovo vescovo voleva imporre ai proprietari ricchi; ${ }^{23}$ e nel 1462 leggiamo che continuava a mantenere contatti commerciali anche con Forte d'Antonio... ${ }^{24}$ La dinamica sociale cambiò l'atteggiamento di un gruppo di patrizi: Dragačić, uomo d'affari, proprietario, leader dei contadini, tutore e procuratore di numerose famiglie - uomo che non perse niente della sua violenza, ma soprattutto, come vedremo, uomo di fiducia delle autorità centrali, pareva sempre di più un personaggio che non era più possibile ignorare.

4. Per capire meglio il capitale sociale che Dragačić riuscì a accumulare, bisogna analizzare più in dettaglio i modi e i discorsi che esprimono conflittualità. L'archivio di Curzola fornisce in effetti dei documenti che permettono allo storico di ricostruire la dimensione quotidiana delle tensioni politiche, che fino a oggi erano conosciute solo tramite gli atti del Senato; questi documenti, esclusivamente processi penali, ci invitano a entrare in un mondo quasi sconosciuto, la vita nelle calli e sulla piazza di Curzola, lasciandoci ascoltare le voci dei protagonisti. Certo, esse erano spesso registrate dal cancelliere del conte; ma non dimentichiamo le numerose petizioni dei protagonisti, la loro conoscenza profonda del diritto locale e la loro capacità di scrivere e di leggere. Questo è vero anche nel caso dei Dragačići il cui talento retorico sarà discusso fra poco. I nostri testi, comunque, passarono tutti tramite un filtro linguistico, la lingua comune a Curzola essendo esclusivamente il čacavo, mentre la versione veneziana costituisce dunque quasi sempre una traduzione. Non possiamo in questa sede trattare il complicato problema linguistico a Curzola, ma riteniamo che i leader politici, patrizi e popolani, viaggiassero regolarmente nello spazio adriatico, andassero spesso a Venezia e fossero senza dubbio capaci di esprimersi bene in veneziano. 
Sottolineiamo, prima di tutto, l'emozionalità e la violenza del discorso. Quando Dragačić tornò da Venezia nell'agosto 1444, i contadini, «che avevano aspettato Zuanino e Francesco come dei» (tamquam Deos), ${ }^{25}$ tutti in arme, gridarono di voler bere il sangue dei patrizi e di farne carne macinata («se facturos carnes de multis aliis et bibere de eorum sanguine»); ${ }^{26}$ alcuni anni più tardi, una donna patrizia promise una ricompensa a chiunque le avesse fornito la carne di Dragačić che voleva mangiare: «una zintildonna de Curzula ha dito che la manzava lire una de le mie carne ruste chi le dessi $\rangle^{27}$ - segno di odio viscerale, della volontà di sradicare il nemico; un odio che da tutte le parti esplose talvolta apertamente, ma che fu canalizzato in un discorso di estrema violenza, che contrasta con la mancanza quasi totale di conflitti sanguinosi.

Bisogna dunque analizzare il linguaggio simbolico di violenza e di conflittualità, con cui si minacciava, si provocava, ma mai fu sparsa una goccia di sangue in un conflitto armato. La paura del sangue, l'ossessione del sangue caratterizza il discorso e gli atti dei Curzolani: il porto di armi era severamente vietato, ma, come abbiamo visto, i contadini giravano sull'isola con le loro spade, coltelli e archi. I patrizi non mancavano nessuna occasione di lamentarsene davanti al conte - ma quello non interveniva, sapendo bene che i contadini non avrebbero mai osato utilizzare armi fatto che avrebbe costituito un atto di ribellione non solo contro i patrizi, ma anche contro il dominio veneziano. Perciò le armi appaiono spesso nei processi, non come oggetti di cui si faceva uso reale, ma come elementi di un discorso che mirava a criminalizzare l'avversario politico agli occhi del dominio veneziano. Lo stesso vale per il sangue sparso con le armi; ma il sangue poteva scorrere anche senza fare ricorso alle armi; il discorso violento si trasponeva non raramente in violenza reale: al culmine dell'affare Dragačić, Francesco di Marino, seguace di Dragačić, incontrò nell'atrio del palazzo vescovile il patrizio Marin Žilković, per decenni un nemico giurato di Dragačić, il quale gli diede di «figlio di putana», colpendolo sul naso «cum maxima effusione sanguinis». ${ }^{28}$ Žilković non cercò di scusarsi, ma dichiarò anzi di avere «aliquam maximam suspitionem.. habitam de eo». Nel maggio 1452 un seguace di Dragačić versò in una rissa il sangue

25. DAZ, AK, 10/13/1, f. 56r

26. DAZ, AK, 10/13/1, f.54r.

27. DAZ, AK, $17 / 32 / 3$, f.30v.

28. DAZ, AK, 10/14/1, f. 22r, 6 novembre 1444. 
di un artigiano; Dragačić non era riuscito a separare i due e fu attaccato dal canto suo dalla moglie dell' artigiano, che lo tirò per i capelli.

La violenza fisica e la forza fisica costituivano un elemento importante nella lotta di potere, ma questo vale di nuovo piuttosto per la dimensione simbolica: Dragačić voleva battersi in duello con Dobroslav Obradović, ma quello rifiutò con le parole: «non sono così stupido di battermi con te» («ego non veni facere questionem quia non sum factus stultus quod velim pugnare tecum»);29 il patrizio preferì dunque evitare il confronto fisico con il giovane leader dei contadini, giustificandolo come atto di intelligenza. Ma è fuori dubbio che i contadini testimoni dell'incidente avessero capito la sconfitta simbolica del patrizio. Nel 1462 un litigio a causa di un terreno a Čara degenerò in una rissa pericolosa, e Francesco Maršić, ricchissimo nipote e erede di un prete corrotto, gettò delle pietre e rincorse Dragačić con la spada snudata. ${ }^{30}$ La violenza fisica si dirigeva anche contro la famiglia dei protagonisti; Dragačić accusò varie volte i suoi nemici di aver battuto sua moglie (1462) o i suoi figli, e questi ultimi sarebbero stati assaliti da una guardia campestre durante la vendemmia. ${ }^{31}$ Osserviamo anche casi di violenza indiretta, come per esempio l'insulto simbolico di Franuša, che sputò davanti ad alcune donne nemiche, parenti della famiglia Obradović e avversarie di Dragačić, delle quali una l'aveva insultata dandole della «putana»; ${ }^{32}$ piccoli incidenti di vicinato, che sembrerebbero insignificanti se non si sapessero gli antecedenti delle persone coinvolte; anzi, il 28 aprile 1458, il patrizio Francesco Obradović accusò il popolare Paolo Glavić di avergli avvelenato quattro galline; il conte incaricò una commissione, che infatti trovò gli animali morti nel cortile del patrizio, procedura che dimostra che prese l'affare molto sul serio. ${ }^{33}$

Occorre anche analizzare la topografia conflittuale: l'istigazione al duello di Dragačić ebbe luogo fuori le mura, nel contado, dunque nello spazio dei contadini dove i patrizi si sentivano in terra nemica; dobbiamo una ottima descrizione di questo sentimento al giudice Mikšić. Dragačić, incoraggiato dai suoi successi, penetrò nello spazio riservato ai patrizi, ov-

29. DAZ, AK, 7/1071, f. 37r.

30. DAZ, AK, 17 Processus doni Marci Radetini cum Antonio Marsich simul cum aliis processibus, f. $38 \mathrm{v}$.

31. DAZ, AK, 17 Processus doni Marci Radetini cum Antonio Marsich simul cum aliis processibus, f. $38 \mathrm{r}$; DAZ, AK, 20/30/12 nota tra f. $12 \mathrm{v}$ e $13 \mathrm{r}$.

32. DAZ, AK, 15/29/4, f. 14v-15r.

33. DAZ, AK, $15 / 29 / 3$, f. $494 \mathrm{v}$. 
vero nella città e più esattamente nella zona vicino alla loggia. La loggia simbolizza e costituisce lo spazio sociale dei patrizi par excellence; qui, a due passi dalla porta di terra, si incontravano i patrizi per giocare a carte, per commentare le persone che entravano, per concludere affari, per fare politica. Dragačić circolava anche in vicinanza di questo luogo simbolico e sfidava i patrizi nel loro ultimo spazio di ritiro; la tensione esplose nel febbraio 1456: i gastaldi di Curzola stavano portando un contadino incatenato al carcere e Dragačić sbarrò loro il passaggio chiedendo l'estradizione di questo uomo onesto, come sosteneva; i patrizi nella loggia saltarono in piedi, alcuni uscirono; ma si infuriarono quando Dragačić toccò uno degli sbirri. Questa trasgressione, il contatto fisico, accelerò la degenerazione della situazione. Dragačić non rimase solo, ma da tutti i canti affluirono i popolani in suo aiuto.

5. Benché gli atti di violenza fisica possano sembrare più spettacolari, i conti veneziani non sottovalutarono il peso della violenza verbale: non a caso il 13 gennaio 1443 il conte obbligò i leader dei patrizi e dei popolani a presentarsi ogni sera nel palazzo, perché «predicti contaminabant homines habitantes in hac insula ad partialitates et odia qui incepti denuo sunt postquam aliqui eorum venerunt de Veneciis»; ;4 il 7 novembre 1446 il conte proibì a Zuanin e a Giorgio Grupšić «alter alterum iniuriare verbis nec iniurare factis»; l'8 gennaio 1447 il milite Nicolò, guardia di Curzola, comminò a Dragačić e a Marin Baronić una ammenda per ingiurie reciproche. Annotiamo a margine che, in pochi giorni, il conte punì tutta una serie di Curzolani per tali offese. ${ }^{35}$ E nel 1458 il conte minacciò Zuanin e Forte d'Antonio di farli incarcerare, se non avessero posto fine ai loro litigi. ${ }^{36}$

Il discorso inerente al conflitto conosceva diversi livelli di intensità, cominciando con le piccole risse quotidiane non esplicitamente motivate da differenze politiche: il 9 settembre 1447 Nika Petrović accusò Zuanin di aver venduto a Čara della carne, contro l'ordine del conte, e di averlo insultato come «filu latru» (un probabile riferimento a tracce della lingua dalmata a Curzola). ${ }^{37}$ L'incidente davanti alla loggia invece provocò lo scambio di accuse molto più gravi. «Sempre siete stati i nemici dei nobili di questo luogo e sempre avete voluto distruggerli», «voi volete sostenere i

34. DAZ, AK, 10/14/1, f. $173 \mathrm{v}$.

35. DAZ, AK, $11 / 17 / 3$, f. $2 \mathrm{v}-4 \mathrm{r}$.

36. DAZ, AK, $15 / 28 / 1$, f. 81 r.

37. DAZ, AK, 11/17/2, f. 10v. 
ladri, voi avete fatto una casa di sangue di questo popolo che avete ingoiato»; «non devi impedire la giustizia, devi permittere la giustizia» - queste furono le accuse dei patrizi contro Dragačić, i quali esprimono in tal modo la loro frustrazione di fronte al leader dei contadini che godeva dell'appoggio incondizionato della popolazione rurale e che era riuscito a ridurre a un minimo le competenze giuridiche dei patrizi. ${ }^{38} \mathrm{Ma}$ il confronto più violento ebbe luogo nel villaggio natale di Dragačić, a Čara, nell'agosto del 1444, quando il giudice Mikšić cercò di far arrestare Dragačić. Quello lo salutò all'entrata del villaggio con le parole «Quis est ille filius putane qui vult prendere nos?». "Quis est ille becus qui vult prendere me, veniat ad capiendum me!». Quando il giudice emanò l'ordine di arresto, Dragačić lo afferrò e lo derise dicendo: «Fac unam proclamationem publice, quia nemo sit tantum ausus quod veniat ad capiendum aliquem de nobis, nisi vult mori»; una minaccia che fu sottolineata dalle spade snudate dei suoi seguaci. E quando i pochi partigiani dei patrizi si accinsero ad avvicinarglisi, prese il suo arco e disse: «Si ad me veneritis, nemo de vobis reverteretur». ${ }^{39}$ Minacce serie, ma minacce che non si indirizzavano contro il dominio veneziano, ma contro il giudice nobile; il giudice ne fu impressionato, ma si ritirò anche davanti a un'arma ancora più pericolosa, i due ducali che Dragačić teneva nella mano e che annunciavano l'intervento del Doge stesso nell'affare Franuša.

Le parole possono uccidere, questo lo sapevano anche i Curzolani, e erano coscienti che niente era più pericoloso dell'accusa di alto tradimento. Patrizi e popolani si scambiavano reciproche accuse davanti alle autorità veneziane, rimproverando al partito nemico cospirazioni e progetti di offendere e di assassinare il conte. I popolani denunciarono il patrizio Mateo Očičić, che aveva rifiutato di tagliare legna per il conte, dicendo che questo sarebbe lavoro da contadino e che il conte andrebbe spedito in catene a Venezia. La pena fu severa; dal canto suo, Očičić accusò Dragačić di averlo insultato e picchiato sulla piazza di Curzola. ${ }^{40} \mathrm{Al}$ culmine del conflitto nell'affare Franuša, i patrizi sparsero la voce che Dragačić, offeso dal conte, avrebbe fatto intervenire i Catalani, all'epoca in guerra aperta con la Serenissima.$^{41}$ Dopo l'incidente davanti alla loggia, il patrizio Antonio Sai-

38. DAZ, AK, 14/26/2, f. 10r-11v.

39. DAZ, AK, $10 / 13 / 1$, f. $54 \mathrm{r}-55 \mathrm{v}$.

40. DAZ, AK, 15/29/3, f. 514r 18 ottobre 1458.

41. DAZ, AK, 10/13/1, f. 57r. 
mier attestò che Dragačić aveva minacciato di uccidere il conte..$^{42}$ Dragačić non esagerava quando in varie occasioni parlò di «nemici mortali». ${ }^{43}$

6. L'aggressione verbale non rimaneva monopolio dei maschi; Franuša sapeva difendersi contro i suoi concittadini (e più spesso ancora contro le sue concittadine) che la insultavano non raramente; sotto l'archivolto di donna Goia Franuša replicò a un insulto di un certo Melchiore con le parole «bastar de fiol, fiol de una putana, che me credi tu far» ${ }^{44}$ (maggio 1459).

Il conflitto politico-sociale non si limitava però allo scambio di parole ingiuriose; alle radici delle tensioni stava l'interpretazione del diritto locale, della costituzione, degli statuti di Curzola. Malgrado la violenza fisica e verbale degli avversari principali, i leader patrizi e popolani si mostrarono ottimi conoscitori del diritto, dotati di una impressionante capacità retorica. Già molto presto Zuanin Dragačić stupiva i conti veneziani e i patrizi curzolani con il suo dono retorico; nel 1445 si difese abilmente contro le accuse di Forte d'Antonio e dei «citadini del povolo antigo», riferendosi alle tradizioni dei contadini e all' «antiga usanza», dimostrando di conoscere bene la storia recente dell'isola; si mostrò anche orgoglioso della sua educazione; i seguaci di Forte, i cittadini di Curzola, non avrebbero mai potuto redigere l'accusa contro di lui, perché non sapevano scrivere; lui invece, e suo fratello, erano letterati, suo fratello Zanin scrisse il suo testamento manu propria. Non conosciamo l'origine delle conoscenze giuridiche, storico-politiche e retoriche di Dragačić, ma durante la sua carriera ebbe molte occasioni di raffinare queste sue qualità; non fu un semplice contadino, ma un uomo che già nella sua gioventù andava a Venezia per negoziare con Francesco Foscari; che già da giovane conosceva bene il sistema giuridico veneziano e non esitò mai a portare il suo caso davanti agli auditori nuovi nella Serenissima. Il vocabolario grossolano della gioventù si andava lentamente trasformando in una retorica fiorita di cui diamo due esempi, tratti da un appello contro la sentenza del conte Piero Soranzo e da un processo contro gli abitanti del villaggio di Lumbarda che avevano distrutto la sua casa. Nel primo caso, del settembre 1448, Dragačić critica l'atteggiamento del conte Soranzo, che aveva forzato i giudici di condannarlo nella causa Franuša:

42. DAZ, AK, 14/26/2, f. 11v.

43. DAZ, AK, 17 Processus doni Marci Radetini cum Antonio Marsich simul cum aliis processibus, f. $38 \mathrm{v}$.

44. DAZ, AK, 15/29/4, f. 14v-15r. 
E fici reverentia de la bereta e levosse suxo e comenzò rengar e avocar contra de mi e rengò un gran pezo digando che mi meritava punimento e ogni mal che li pareva dir de mi, cosa de uno rector mai alo mondo non fo vista ni aldida, che zudexi davanti el qual se dumanda raxon e justitia avocarsi per la parte mostrando manifeste la soa oppinion; e quando io li volsi responder, non me lassò dir parola, ma subito intrò in cancelleria. e fici sonar campana $\mathrm{e}$ vene fora cum cancelier e fici lecer sententia e publicola. ${ }^{45}$

Quattordici anni più tardi, Dragačić fornisce un'altra prova del suo dono retorico:

supplico ala vostra santa Justitia che per intuito e vigor de quello vi piaza condanar e sententiar quelli de lo caxal de Lumbarda chomo arobadori per forzo, violentia, vituperio e despexio de la santa vostra justitia e de mi servidor vostro loqual soto le ale e protetion vostra vivo, pagar ducati xxv d'oro applicando per la mità al vostro comun altra a mi al qual vien fata robaxion e violentia e questo chomo se fa per tuto l'universo mundo dove le raxon e Justitia azo che reami, provintie, terre, luogi e casali possano e vagliano star in paxi e seguri perché altramente a nessun de nuy non besognar tegnir in caxe nostre tanto quanto val un denaro picolo ni valea caxe nostre cossa alchuna, se la vostra Justitia non provede e se queste inconvenientie e assassinamenti li passano impunidi etc. ${ }^{46}$

Le dichiarazioni di lealtà e di fedeltà allo Stato veneziano costituiscono un vero e proprio filo rosso nella retorica di Dragačić attraverso gli anni; questi abilmente paragona la giustizia veneziana ad una realtà nuova nella vicinanza di Curzola, l'Impero ottomano, invocando la «Turchia over in luogo dove non se tien ni raxon ne justitia alchuna». ${ }^{47}$ I suoi avversari patrizi si videro costretti ad utilizzare dal canto loro la stessa retorica, ma soprattutto derisero la pretesa di un non-patrizio nell'interpretazione degli statuti. Infatti i patrizi percepivano questo atto come un loro diritto riservato, esclusivo; ed il fatto che Dragačić invocasse spesso questo codice pareva una intrusione in uno spazio giuridico-sociale protetto. Questa guerra di lettura e d'interpretazione degli statuti è un altro filo rosso nella vita di Dragačić: già nel corso del suo primo grande processo contro Marco Goriglavic, i capitoli 123 e 140 - che proibiscono ai Curzolani di difendere gli interessi di forestieri contro altri Curzolani - erano al centro del litigio. ${ }^{48}$

45. DAZ, AK, 12/20/2, s.p.

46. DAZ, AK, 17/32/3, f. 22 r.

47. Ibidem.

48. DAZ, AK, 7/9/1-2, f. 160r. 
Quando nel 1441 Zanin Dragačić revocò un contratto di matrimonio, si richiamò «al nostro statuto». ${ }^{49}$ Abbiamo già raccontato come il conte cercasse nel manoscritto degli statuti un paragrafo contro Zuanin Dragačić. Nel 1462 Dragačić recita allo scopo tutta una serie di paragrafi:

la lege de Curzula confermada per la nostra glorioxa Signoria de Veniexia non vol che uno testimonio fede degno dizi la lege sia agetà, salvo per fina grossi /12/ e dui testimonii fede degni per fina pp 10 e de diexe pp in su non vol che alchuna testimonianza vaglia senza instrumento over scritura de propria man del debitor... Item altra lege sta a capitoli /16/ la qual dixe che alchuno testimonio non debia esser ageta, sel non serà sta chiamato saltem per una de le parte per testimonio specialiter etc... Item oppono a tuti li testimonii per Zugno contra de mi produti per esser mie inimicissimi e perché instessi se hano messi avanti e prima bistole (?) de Jacomo per haver litigado cum mi davanti el vostro precessor e si me è stà condannado in certi denari per li qual per esser pagato io fexi intrometter uno suo caxamento et vender per modo che me ha manazato più volte de amazar si che concludo che non pò vi dir esser tolto per testimonio contra di me perché nostro Statuto a capituli /36/altro a capituli $/ 37 /$ non vol che inimicho manifesto sia tolto per testimonio. ${ }^{50}$

I patrizi derisero questa giurisprudenza da parte di un non-patrizio: «ser Zuhanino lo qual aliega molti argumenti chomo savio e intelligente statuti, le leze, usanze e molti altri beli testi de le lege chomo savio e si ha posto uno argumento uno pocho vitiosamente e si domanda a me Mateo tereno e le pertinentie mie de loqual tereno» - esclamò, nel giugno 1464, il patrizio Antonio Saimier. ${ }^{51}$ Una guerra retorica dunque, ma tutte queste parole non si indirizzavano al campo avversario, ma alla potenza che nel nostro racconto finora è rimasta nell'ombra: la Repubblica di Venezia.

7. Benché abbiamo già accennato a varie riprese alla presenza veneziana sull'isola, dobbiamo strutturare le nostre osservazioni: Venezia era presente in due ipostasi diverse, la prima incarnata dal conte, e poi come metropoli lontana dove risiedono le autorità centrali, il doge e gli auditori novi. A Curzola la residenza permanente di Veneziani non era la regola - al contrario, il conte si trovava spesso solo di fronte a una società isolana di 4-5.000 persone. Non vi erano soldati o ufficiali veneziani; la difesa e tutta l'amministra-

49. Ivi, f. 182 r.

50. DAZ, AK, 17 Processus doni Marci Radetini cum Antonio Marsich simul cum aliis processibus, f. $37 \mathrm{v}-38 \mathrm{r}$.

51. DAZ, AK, 18/33712, f. 50v 
zione venivano gestite direttamente dalla popolazione locale. La posizione del conte era forte e fragile nello stesso tempo, e molto dipendeva dalla sua personalità. Abbiamo già visto che la popolazione misurava il tempo in categorie di potere, cioè il reggimento dei rispettivi conti. Ai suoi occhi il conte costituiva la persona principale di riferimento; ma dal nostro racconto risulta chiaramente che l'autorità del conte non rimase incontestata. Patrizi e popolani cercavano di attirarlo nel loro campo e di strumentalizzarlo nelle lotte interne dell'isola. Gli isolani osservavano attentamente ogni passo del conte e lo giudicavano secondo le loro preferenze politiche. Un conte incauto che prendesse esplicitamente partito per uno dei campi, metteva in pericolo il suo prestigio, ma anche tutto il sistema del buon governo. Un conte che sostenesse la causa dei patrizi non aveva niente da dire nello spazio rurale: gli esempi più drastici in tal senso sono Marco Gradenigo e Piero Soranzo, tutti e due alleati dei patrizi nella causa Franuša.

Quanto fosse fragile la loro posizione viene illustrato da alcuni esempi desunti dalla biografia politica di Dragačić. Torniamo un'ultima volta all'inizio della sua carriera; nel settembre 1441 il conte limitò la libertà di movimento di Dragačić e il cancelliere registrò negli atti che il giovane leader contadino reagì «verbis arrogantibus et minatoriis dicens "videte violentiam quam facit mihi dominus comes"»; tenne a non minacciare la persona del conte, ma solo i giudici, tutti nobili curzolani, e il notaio. ${ }^{52}$ Sempre nell'assenza del conte, aggiunse poco dopo, «el no avrà tanta força lo conte e li çudixi poner me in carceribus», oltraggio grave che il notaio annotò nel registro invocando la testimonianza di ser Scimon Nicholich e del plazarius Marco. ${ }^{53}$ Il conte fece subito condannare Dragačić alla pena enorme di 100 ducati, ${ }^{54}$ ma ottenne solo l'effetto che Dragačić tenesse un grande discorso davanti ai contadini adunati nel campo di Polud; lo stesso giorno provocò Obradovic al duello: "ser Obradi, vos venistis me aggressum hic, si quis vestrum vul (sic) facere mecum cum armis, veniat foras» $>{ }^{55}$ Giorgio Grupšić, esponente radicale del partito dei patrizi, si lasciò trasportare dalla sua ira a causa delle adunanze contadine e proclamò che «misser lo conte che forsi non anderà cum la testa de qui zoè cum la vita e se l'anderà, serà vergognado da la Signoria» ${ }^{56}$ E non dimentichiamo il caso di Marko Očičić, che minacciò il 
conte «Fortassis nos mittemus eum Venetias victum». ${ }^{57}$ La persona del conte poteva esser criticata, e i Curzolani non si contenevano, ricorrendo a espressioni aspre; ma sempre comunque distinguendo bene e con molta prudenza tra il conte e lo Stato veneziano. Mai quest'ultimo fu oggetto di commenti negativi o di disprezzo. I Curzolani in altre parole si rendevano conto della differenza tra la protesta contro il malgoverno di un individuo e il tradimento. Con l'eccezione di Giorgio Grupšić, nessun Curzolano osava articolare sentimenti di opposizione fondamentale; al contrario, Venezia e le autorità centrali costituivano una necessità, l'elemento indispensabile per l'equilibrio politico nell'isola; infatti l'odio politico dei partiti curzolani rischiava di paralizzare l'amministrazione e la giustizia; e senza mediatore il conflitto sarebbe degenerato in guerra civile, con massacri nello stile della ribellione popolare di Lesina nel 1510.

Se una guerra vi fu a Curzola, è stata una vera e propria guerra di processi. I processi davanti al conte, davanti agli auditori novi, gli appelli fino al doge stesso - queste erano le armi nella lotta politica a Curzola. Ambedue le parti coltivavano le loro rispettive relazioni con il patriziato veneziano, i patrizi preferendo il contatto diretto con i conti, patrizi come loro stessi, i popolani favorendo le autorità centrali. Un Dragačić era una persona ben nota nella corte degli auditori nuovi ${ }^{58}-$ «me ho appellato viva voce ai Signori Aldidori novi $\rangle^{59}-$; un uomo che sapeva usare il sistema giuridico veneziano a suo favore. Nel conflitto davanti alla loggia, la minaccia di andare a Venezia («si ivero Venecias, quod me cognosces») fece tacere i suoi avversari politici, che conoscevano fin troppo bene l'appoggio di cui godeva Dragačić presso Francesco Foscari; «i miei vestiti sono lacerati», urlò il patrizio Antonio Saimier. ${ }^{60}$ Il nome di Venezia bastava per mettere fine a un conflitto. Conosciamo la risposta politica dei patrizi, l'affare Franuša, il furto della capra, e aggiungiamo altri casi: il leader contadino Francesco di Marino accusò nel luglio 1442 il patrizio Matteo Rosen di aver contrabbandato grano albanese da Durazzo. ${ }^{61}$ I più spettacolari processi per contrabbando, nei quali furono coinvolti patrizi di rilievo come Forte d'Antonio, furono quasi tutti iniziati da popolani. Furto di capre, contrabbando: a ognuno il suo delitto economico.

57. DAZ, AK, 10/14/1, f. 30v.

58. DAZ, AK, 12/13/1, f. 19r-v; f. 56r.

59. DAZ, AK, 12/20/1, s.p.

60. DAZ, AK, $14 / 26 / 2$, f. $11 \mathrm{v}$.

61. DAZ, AK, 10/13/1, f. 4v. 
Torniamo, per finire, ancora una volta al livello microstorico per soddisfare la nostra curiosità: come è finita la storia d'amore di Dragačić e di Franuša? Cos'è successo al loro figlio?

Il 28 luglio 1456, Zanin Dragačić fece testamento, lasciando a Franuša una casa e quattro vigne, ed al figlio di Franuša una casa - un gesto di affetto che sembra confermare l'immagine di una relazione stabile. Ma quattro anni più tardi Zuanin Dragačić citò Franuša davanti al conte chiedendole 1'affitto degli otto anni in cui aveva vissuto come locataria in casa sua: ${ }^{62}$

comparo mi Zuhanin fo de ser Franescho domandando raxon et justitia contra Franussa fiola fo de Radassin de Stagno cum sit che la dita steti in caxa mia a fito cercha anni oto chomo sola ha confessado in presentia del vostro lucido conspeto che le sta in caxa mia e che nin me ha pagato el mio affito may per laqual cossa supplico ala Magnificencia vostra che vi piaza sententiar dita Franussa a mi dar e pagar per li prediti affiti de caxa mia per anni oto ducati /16/ zoe ducati dui al anno.

Il 25 ottobre Franuša replicò, e lasciamo a lei la parola:

non denego may anzi conciedo esser sta in caxa soa ma non ad affito però chel me era tegnudo dar la caxa chomo quello el qual si me havea fato descargar de caxa de mio padre et/de ogni ben per lui privata mosso de pietà el me mandò pregando più e più volte che dovessi intrar in caxa soa, intrai et morando in caxa soa;

aggiunse che il suo ex amante non le aveva mai consegnato la casa e le vigne che suo fratello Zanin le aveva lasciato in eredità. E conclude con una controaccusa:

el me mossi sta lite volendo me far paura e pensando che per non saver dir le mie raxon perischa; concludo non haver tolto la caxa soa may a fito, anzi pregato per lui chomo voglio provar, sun intrada in caxa sua et insida fora de caxa soa cum so voler e sun passati ora may anno e mexi et may non dimandò niente, avixando le signorie vostre che passato uno anno non se puol dimandar may niente per fito de caxa offerendome provar ut supra per laqual cossa prego la Signoria vostra che me absolvit de la sua dumanda...

Franuša sapeva difendere i suoi diritti, era in grado di sopravvivere in un mondo dominato da uomini. Non si era mai sposata, e a suo figlio aveva dato il nome orgoglioso di Ettore - il figlio di un nemico del suo ex aman- 
te si chiamava Paride. L'umanesimo è entrato in tal modo nel linguaggio politico dei contadini di Curzola. Ettore appare spesso nei registri di «criminalia», che, in quanto figlio illegittimo, non poté approfittare dell'ascesa sociale di suo padre e dei suoi figli legittimi. Franuša e suo figlio rimasero dunque ai margini della società curzolana, Zuanin Dragačić invece continuò la sua carriera politica e commerciale.

La storia di Dragačić e di Franuša sembra illustrare le conclusioni del contributo precedente dalla penna di Ermanno Orlando. Infatti, «la negoziazione e il compromesso» (Orlando p. 60) costituiscono il filo rosso dell'atteggiamento degli ufficiali veneziani di fronte a una realtà sociale $\mathrm{e}$ giuridica molto complessa. Dallo studio del nostro caso emergono i limiti del potere del governatore veneziano, l'importanza della sua personalità che influenzava fortemente l'atteggiamento delle varie frazioni locali che oscillavano tra deferenza e provocazione aperta dell'autorità del governatore - ma non dello Stato veneziano in genere. Si costata, infatti, una costellazione di potere che includeva patrizi e popolani locali, le autorità centrali e i rappresentanti locali di Venezia che non seguivano sempre una politica coerente; questo vale anzitutto per la posizione del rettore che dipendeva dagli interessi e preferenze rispettivi dell'ufficiale che sosteneva ora i richiami dei popolani ora gli argomenti dei patrizi. Emerge anche dalla nostra storia quanto fosse fluida la distinzione tra i ceti locali. Certo, patrizi e popolani costituivano comunità distinte, definite come tali negli Statuti dell'isola. Comunque, questa barriera costituzionale non impediva ai popolani di aderire al "partito" patrizio o, come dimostra il caso di Dragačić, di mantenere legami commerciali ed economici con avversari politici. Sarebbe erroneo pensare la società curzolana in categorie di classe troppo rigide e immutabili. Per riprendere le parole di Ermanno Orlando, la dinamica dialettica non caratterizza soltanto la relazione tra Venezia e la sua periferia, ma anche le costellazioni di potere all'interno della società locale. L'approccio microstorico rafforza in questa prospettiva i risultati di un'analisi più generale, basata su documenti che riflettono il punto di vista delle autorità centrali, ma aggiunge carne alle ossa secche delle decisioni del Senato o delle petizioni (capitula) dei sudditi veneziani. 